\title{
Induction Chemotherapy with Gemcitabine and Cisplatin Followed by Simultaneous Integrated Boost-Intensity Modulated Radiotherapy with Concurrent Gemcitabine for Locally Advanced Unresectable Pancreatic Cancer: Results from a Feasibility Study
}

\author{
Sang Myung Woo, MD, PhD ${ }^{1}$ \\ Min Kyeong Kim, BS² \\ Jungnam Joo, $\mathrm{PhD}^{3}$ \\ Kyong-Ah Yoon, PhD ${ }^{4}$ \\ Boram Park, MS ${ }^{3}$ \\ Sang-Jae Park, MD, PhD ${ }^{1}$ \\ Sung-Sik Han, MD, PhD' \\ Ju Hee Lee, MD, PhD ${ }^{1}$ \\ Eun Kyung Hong, MD, PhD ${ }^{1}$ \\ Yun-Hee Kim, $\mathrm{PhD}^{5}$ \\ Hae Moon, MD \\ Sun-Young Kong, MD, PhD2,7 \\ Tae Hyun Kim, MD, PhD' \\ Woo Jin Lee, MD, PhD'
}

*A list author's affiliations appears at the end of the paper.

\author{
Correspondence: Woo Jin Lee, MD, PhD \\ Center for Liver Cancer, National Cancer Center, \\ 323 Ilsan-ro, Ilsandong-gu, Goyang 10408, Korea \\ Tel: 82-31-920-1612 \\ Fax: 82-31-920-2798 \\ E-mail: lwj@ncc.re.kr \\ Co-correspondence: Tae Hyun Kim, MD, PhD \\ Center for Liver Cancer, National Cancer Center, \\ 323 Ilsan-ro, Ilsandong-gu, Goyang 10408, Korea \\ Tel: 82-31-920-1725 \\ Fax: 82-31-920-2798 \\ E-mail: k2onco@ncc.re.kr
}

Co-correspondence: Sun-Young Kong, MD, PhD Department of System Cancer Science, Graduate School of Cancer Science and Policy, Center for Diagnostic Oncology, National Cancer Center, 323 Ilsan-ro, Ilsandong-gu, Goyang 10408, Korea Tel: $82-31-920-1735$

Fax: 82-31-920-2798

E-mail: ksy@ncc.re.kr

Received October 16, 2016

Accepted January 3, 2017

Published Online January 19, 2017

*Sang Myung Woo and Min Kyeong Kim contributed equally to this work.

\section{Purpose}

This study assessed the feasibility and compliance of induction chemotherapy with gemcitabine and cisplatin followed by simultaneous integrated boost-intensity modulated radiotherapy (SIB-IMRT) with concurrent gemcitabine in patients with locally advanced unresectable pancreatic cancer.

\section{Materials and Methods}

In this trial, patients received induction chemotherapy consisting of gemcitabine $(1,000$ $\left.\mathrm{mg} / \mathrm{m}^{2}\right)$ and cisplatin $\left(25 \mathrm{mg} / \mathrm{m}^{2}\right)$ on days 1,8 , and 15 of each treatment cycle. Patients were subsequently treated with gemcitabine $\left(300 \mathrm{mg} / \mathrm{m}^{2} / \mathrm{wk}\right)$ during SIB-IMRT. The patients received total doses of 55 and 44 Gy in 22 fractions to planning target volume 1 and 2, respectively. As an ancillary study, digital polymerase chain reaction was performed to screen for the seven most common mutations in codons 12 and 13 of the KRAS oncogene of circulating cell free DNA (cfDNA).

\section{Results}

Forty-four patients were enrolled between 2012 and 2015. Of these, 33 (75\%) completed the treatment. The most common toxicities during induction chemotherapy were grades 3 and 4 neutropenia (18.2\%), grade 3 nausea (6.8\%) and vomiting (6.8\%). The most common toxicities during SIB-IMRT were grade 3 neutropenia (24.2\%) and grade 3 anemia (12.1\%). Ten patients (23\%) underwent a curative resection after therapy. Median overall survival was significantly longer in patients who underwent curative resection (16.8 months vs. 11 months, $p<0.01$ ). The median cfDNA concentration was significantly lower after treatment (108.5 ng/mL vs. $18.4 \mathrm{ng} / \mathrm{mL}, \mathrm{p}<0.001)$.

\section{Conclusion}

Induction chemotherapy with gemcitabine and cisplatin followed by concurrent SIB-IMRT was well tolerated and active.

\section{Key words}

Pancreatic neoplasms, Radiotherapy, Gemcitabine, Cisplatin 


\section{Introduction}

Pancreatic cancer is one of the few cancers for which survival has not improved substantially over the past 40 years [1]. Currently, pancreatic cancer is the fifth leading cause of cancer-related death in Korea [2]. In 2012, 5,403 patients in Korea were newly diagnosed with pancreatic cancer, and 4,778 individuals died of the disease. At diagnosis, 30\% of patients with pancreatic cancer have locally advanced, unresectable stage 3 disease.

The standard of care for locally advanced pancreatic cancer (LAPC) remains poorly defined. Randomized trials [3,4] comparing chemotherapy alone with chemoradiotherapy (CRT) have yielded conflicting results. Although initial chemotherapy is an increasingly utilized option for patients with LAPC, the optimal regimen has not yet been established [5-7]. The combination of gemcitabine with low-dose cisplatin has shown activity in advanced pancreatic cancer [8]. Meta-analyses revealed that the combination of gemcitabine plus platinum resulted in a significant improvement in progression-free survival (PFS) when compared with gemcitabine monotherapy, although overall survival (OS) did not differ significantly $[9,10]$. This regimen may be an acceptable alternative for patients ineligible for gemcitabine plus nabpaclitaxel, FOLFIRINOX or clinical trials [11,12].

The international phase III LAP 07 study, which compared chemotherapy alone with CRT, demonstrated that, although CRT did not improve survival, patients in the CRT arm with non-progressive LAPC after induction chemotherapy had a longer time without treatment and a significantly lower rate of local tumor progression, which could translate into a better quality of life [13]. In one population-based study, $41 \%$ of LAPC patients treated with first-line chemotherapy died without evidence of distant metastases [14]. These results highlight the heterogeneity of LAPC and the importance of locoregional tumor control. Accordingly, "consolidation" CRT may be a viable treatment option for selected patients with locally advanced non-metastatic pancreatic cancer.

Intensity-modulated radiotherapy (IMRT) is a type of radiotherapy (RT) in which radiation beams can be modulated to deliver high doses to the tumor while reducing the dose to surrounding normal tissues [15]. Conceptually, besides the conformal dose distribution, IMRT can exploit the potential biological advantages of accelerated forms of RT, known as simultaneous integrated boost (SIB)-IMRT in which different doses can be delivered to different targets at the same time. Specifically, a higher dose can be delivered to the gross tumor volume (GTV), while a lower dose is simultaneously delivered to areas of subclinical disease. Dosimetric studies have suggested that implementation of IMRTbased RT may result in improvements in patients with pan- creatic cancer [16]. To extend these observations, the present study assessed the compliance and efficacy of induction chemotherapy with gemcitabine and cisplatin, followed by concurrent SIB-IMRT, in patients with LAPC.

\section{Materials and Methods}

\section{Study design and patients}

Patients aged $\geq 18$ years were eligible if they had histologically or cytologically proven, locally advanced pancreatic cancer, an Eastern Cooperative Oncology Group (ECOG) performance status (PS) of 0 to 1 , no evidence of metastatic disease in the major viscera or peritoneal seeding, and had not previously undergone irradiation of the planned field. Resectability was assessed based on a preoperative staging contrast-enhanced computed tomography scan, magnetic resonance imaging, endoscopic ultrasound, and positron emission tomography. Criteria for local unresectability included at least one of the following: long segment occlusion of the mesenteric vein/portal vein, more than 180degree involvement of the superior mesenteric artery or involvement of the hepatic artery or celiac trunk. At least one bi-dimensionally measurable lesion had to be present.

For inclusion, all sites of malignant disease had to be encompassed within a single irradiation field $(15 \times 15 \mathrm{~cm}$ maximum), and all patients had to have radiographically assessable disease. Patients with biliary or gastroduodenal obstruction must have undergone drainage prior to starting treatment. Required laboratory parameters at study entry were as follows: white blood cell count $\geq 1,000 / \mathrm{mm}^{3}$, hemoglobin level $\geq 7.5 \mathrm{~g} / \mathrm{dL}$, platelet count $\geq 100,000 / \mathrm{mm}^{3}$, creatinine level $\leq 3.0 \mathrm{mg} / \mathrm{dL}$, and total bilirubin level $\leq 3.0$ $\mathrm{mg} / \mathrm{dL}$ (patients with elevated bilirubin due to obstruction had to be stented and their bilirubin level had to be $\leq 3.0$ $\mathrm{mg} / \mathrm{dL}$ prior to study entry).

In this prospective clinical trial, patients received induction chemotherapy consisting of gemcitabine $\left(1,000 \mathrm{mg} / \mathrm{m}^{2}\right)$ and cisplatin $\left(25 \mathrm{mg} / \mathrm{m}^{2}\right)$ as intravenous infusions on days 1,8 , and 15 of each treatment cycle (Fig. 1). Patients were subsequently treated with gemcitabine alone $\left(300 \mathrm{mg} / \mathrm{m}^{2}\right)$ as 30-minute intravenous infusions once weekly during SIBIMRT, starting within 3 weeks of completing two cycles of induction chemotherapy. If patients did not progress distantly following induction chemotherapy, SIB-IMRT was started 3-4 weeks after the end of induction chemotherapy.

For RT planning, patients were placed in the treatment position (generally, supine with arms above the head) and immobilized using an arm-up holder to improve setup 
Before chemotherapy

Test

$\mathrm{CBC}$, liver function test, renal function test, EUS-guided fine needle biopsy, CT, MRI CA 19-9 (PET, CEA, if necessary)
After SIB-IMRT

Test

CBC, LFT, CA 19-9, CT

(CEA, if necessary)

$\begin{array}{lccccc}\begin{array}{l}\text { Blood sample } \\ \text { (blood sampling } \\ \text { and storage) }\end{array} & \downarrow & & & & \\ & \text { D1 } & \text { D8 } & \text { D15 } & \text { D22 } \\ \text { GEM }\left(1,000 \mathrm{mg} / \mathrm{m}^{2}\right) & \uparrow & \uparrow & \uparrow & \\ \text { Cisplatin }\left(25 \mathrm{mg} / \mathrm{m}^{2}\right) & \uparrow & \uparrow & \uparrow & \end{array}$

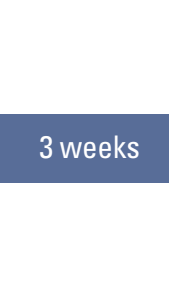

Blood sample

(blood sampling and storage)

SIB-IMRT

[PTV1: $55 \mathrm{~Gy} / 22 \mathrm{Fx} / \uparrow \uparrow \uparrow \uparrow \uparrow \uparrow \uparrow \uparrow \uparrow \uparrow \uparrow \uparrow \uparrow \uparrow \uparrow \uparrow \uparrow \uparrow \uparrow \uparrow ~ \uparrow \uparrow$

PTV2: $44 \mathrm{~Gy} / 22 \mathrm{Fx}]$

$\operatorname{GEM}\left(300 \mathrm{mg} / \mathrm{m}^{2}\right) \quad \uparrow \quad \uparrow \quad \uparrow \quad \uparrow \quad \uparrow \quad \uparrow$

Fig. 1. Treatment schema, showing induction chemotherapy with GEM and cisplatin, followed by GEM-based SIB-IMRT. $\mathrm{CBC}$, complete blood count; EUS, endorectal ultrasound; $\mathrm{CT}$, computed tomography; MRI, magnetic resonance imaging; CA 19-9, carbohydrate antigen 19-9; PET, positron emission tomography; CEA, carcinoembryonic antigen; LFT, liver function test; GEM, gemcitabine; SIB-IMRT, simultaneous integrated boost-intensity modulated radiotherapy; PTV, planning target volume.

reproducibility. Computed tomography (CT) images were acquired over 10 respiratory phases at slices 2.5 -mm-thick under shallow respiration using a four-dimensional CT simulator (Light-Speed RT, GE Healthcare, Waukesha, WI). All CT images were transferred to a treatment planning system (Eclipse, ver. 8.0, Varian Medical System, Palo Alto, CA), and contours for targets and organs at risk were drawn. The GTV included all detectable tumors, as determined by CT. The internal target volume (ITV) was obtained by summing the GTVs of all respiratory motion phases, and the clinical target volume (CTV) included the ITV and the volumes of regional lymph nodes, including the pericholedochal, celiac, and pancreaticoduodenal nodes. Planning target volumes 1 (PTV1) and 2 (PTV2) consisted of the ITV plus 3-5 mm margins in all directions and the CTV plus 5-7 mm margins, respectively. RT planning was performed using five coplanar or non-coplanar intensity modulated beams of $6 \mathrm{MV}$ photons and the prescribed total doses to the PTV1 and 2 were 55 Gy and $44 \mathrm{~Gy}$, respectively, each in 22 fractions. The treatment was designed so that at least $95 \%$ of the PTV would receive $100 \%$ of the prescribed dose, with the maximum dose to the spinal cord not exceeding $45 \mathrm{~Gy}$. The absolute volumes of the esophagus and stomach that received at least 55 Gy were $\leq 2 \mathrm{~cm}^{3}$ each, and the absolute volumes of the small and large intestines that received at least 50 Gy were $\leq 2 \mathrm{~cm}^{3}$ each. Patients with sufficient tumor regression subsequently underwent pancreatic resection 6 to 8 weeks after treatment.

Imaging results during treatment were interpreted and decisions regarding SIB-IMRT or surgery made at multidisciplinary meetings. Upon completion of the protocol therapy, patients were followed up every 3 months for 2 years by CT or magnetic resonance imaging of the chest, abdomen, and pelvis. Response to the protocol therapy was assessed using the Response Evaluation Criteria In Solid Tumor criteria ver. 1.1. Toxicity was scored according to the National Cancer Institute Common Terminology Criteria for Adverse Events ver. 4.1. Data collected for all patients who underwent surgery included the type and duration of surgery, and whether they had undergone vascular resection and/or reconstruction.

All patients had to provide written informed consent before registration, and the trial protocol was approved by the Ministry of Food and Drug Safety in Korea and the Institutional Review Board of the National Cancer Center, Korea (NCCCTS-11-567, ClinicalTrials.gov identifier: NCT01593475). This study was partly sponsored by Dong-A ST, Korea, as well as by grants from the National Cancer Center, Korea.

\section{Cell-free DNA and KRAS mutation analysis}

Cell-free DNA (cfDNA) was extracted from $0.8 \mathrm{~mL}$ of serum using QIAamp Circulating Nucleic Acid Kits (Qiagen, Hilden, Germany) and quantified using Qubit dsDNA HS Assay Kits (Invitrogen, Eugene, OR). As an ancillary study, digital PCR was performed using a QX200 KRAS Screening Multiplex Droplet Digital PCR System (Bio-Rad Laborato- 
ries, Hercules, CA), which covers the mutation sites G12A, G12C, G12D, G12R, G12S, G12V, and G13D. The results were analyzed with the QuantaSoft software (Bio-Rad Laboratories, Pleasanton, CA).

\section{Statistical analysis}

The primary endpoints of this study were the feasibility of and compliance with induction chemotherapy with gemcitabine and cisplatin followed by SIB-IMRT for patients with locally advanced unresectable pancreatic cancer. Because approximately $20 \%$ of patients with locally advanced disease develop early distant metastasis $[4,17]$, it was expected that at least $80 \%$ of all patients would be eligible for SIB-IMRT upon completion of induction chemotherapy. The null hypothesis set the true compliance rate of patients eligible for SIB-IMRT at $\leq 60 \%$. For the study to have a power of $80 \%$ when an experimental arm shows a compliance of $\geq 80 \%$, the required number of evaluable patients was calculated to be 24 at a one-sided type I error rate of $5 \%$. If $10 \%$ of patients are lost to follow-up and 20\% develop distant metastases after induction chemotherapy, then a total of 44 eligible patients would be required to show a significant effect of treatment.

Fisher exact test, Pearson's chi-square test, the Student's t test and the Mann-Whitney test were used when appropriate to identify significant differences in demographic and clinical characteristics. Statistical significance was set at a p-value of less than 0.05. All analyses were performed using the SAS statistical ver. 9.3 and $R$ ver. 3.3.1 and reported p-values are two-sided.

\section{Results}

\section{Patient and primary tumor characteristics}

Forty-four patients consented to participate and were enrolled in this study between March 2012 and January 2015. The patients consisted of 19 males and 25 females with a median age of 67 years, and with a median follow-up period of 13.1 months (range, 4.2 to 41.9 months). Of these 44 patients, nine had an ECOG PS of 0 and 35 had an ECOG PS of 1 . Table 1 summarizes the demographic and clinical characteristics of these patients.

\section{Treatment administration and toxicity}

All 44 patients were started on induction chemotherapy, with $39(89 \%)$ completing the induction treatment regimen.
Table 1. Baseline characteristics

\begin{tabular}{lc} 
Characteristic & No. $(\%)$ \\
Total No. of patients & 44 \\
Age, median (IQR, yr) & $67(57.5-71)$ \\
Sex & \\
$\quad$ Male & $19(43)$ \\
$\quad$ Female & $25(57)$ \\
Tumor size (longest diameter), & $3.6(3.1-4.5)$ \\
median (IQR, cm) & \\
Tumor location & \\
$\quad$ Head & $25(57)$ \\
$\quad$ Body and tail & $19(43)$ \\
Pretreatment CA 19-9, & $267.5(64-773)$ \\
median (IQR, U/mL) & \\
Pretreatment CEA, & $4.25(2.15-7.4)$ \\
median (IQR, U/mL) & \\
ECOG performance status & $9(21)$ \\
0 & $35(79)$ \\
\hline
\end{tabular}

IQR, interquartile ranges (Q1-Q3); CA 19-9, carbohydrate antigen 19-9; CEA, carcinoembryonic antigen; ECOG, Eastern Cooperative Oncology Group.

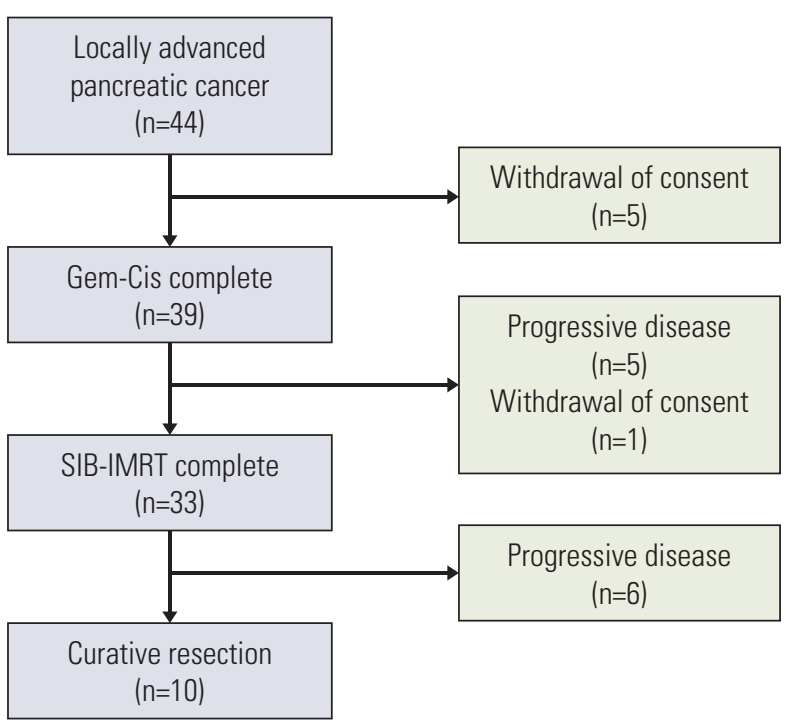

Fig. 2. Flow of patients through the protocol treatment. Gem-Cis, gemcitabine plus cisplatin; SIB-IMRT, simultaneous integrated boost-intensity modulated radiotherapy. 
Table 2. Grade 3 adverse events in $>5 \%$ of patients and all grade 4 adverse events

\begin{tabular}{|lcc}
$\begin{array}{l}\text { Toxicity } \\
\text { TInduction chemotherapy }(\mathbf{n}=44)\end{array}$ & Grade 3 & Grade 4 \\
\hline Hematologic & & \\
\hline Neutropenia & $7(15.9)$ & $1(2.2)$ \\
\hline Thrombocytopenia & $7(15.9)$ & - \\
\hline Anemia & $4(9.1)$ & - \\
\hline Non-hematologic & & \\
\hline Nausea & $3(6.8)$ & - \\
\hline Vomiting & $3(6.8)$ & - \\
\hline SIB-IMRT (n=33) & & \\
Hematologic & & - \\
\hline Neutropenia & $8(24.2)$ & - \\
\hline Thrombocytopenia & $2(6.1)$ & - \\
\hline Anemia & $4(12.1)$ & - \\
\hline Non-hematologic & $2(6.1)$ & \\
\hline Cholangiohepatitis & & \\
\hline
\end{tabular}

Values are presented as number (\%). SIB-IMRT, simultaneous integrated boost-intensity modulated radiotherapy.

After two cycles, five (11\%) patients showed distant progression, precluding SIB-IMRT (Fig. 2). One additional patient withdrew consent prior to starting SIB-IMRT. Thus, 33 patients $(75 \%)$ completed the entire treatment protocol, which was a significantly higher percentage than the $60 \%$ expected ( $\mathrm{p}=0.028$ ), with six patients progressing locally or distantly after SIB-IMRT. All patients completed SIB-IMRT without interruption. The average relative dose intensities of gemcitabine and cisplatin during induction chemotherapy were $81.4 \%$ and $83.2 \%$, respectively. The average relative dose intensity of gemcitabine during SIB-IMRT was $72.5 \%$.

Table 2 summarizes the safety results. The most common toxicities during induction chemotherapy were grades 3 and 4 neutropenia $(18.2 \%)$, grade 3 nausea $(6.8 \%)$, and grade 3 vomiting $(6.8 \%)$. The most common toxicities during SIBIMRT were grade 3 neutropenia (24.2\%) and grade 3 anemia $(12.1 \%)$. Grade 3 and 4 gastrointestinal toxicities were not reported. There were no treatment-related deaths, and no late radiation toxicities such as gastrointestinal bleeding or duodenal ulcer were observed.

\section{Efficacy}

The response rate was $34 \%$ and the disease control rate was $64 \%$ after completion of the entire treatment protocol. The 34 patients with measurable carbohydrate antigen 19-9 (CA 19-9) concentrations at both baseline and after treatment showed a significantly lower median CA 19-9 level after
(25 U/mL; interquartile range [IQR], 6.8 to $135 \mathrm{U} / \mathrm{mL}$ ) than before $(267.5 \mathrm{U} / \mathrm{mL}$; IQR, 64 to $773 \mathrm{U} / \mathrm{mL})$ treatment $(\mathrm{p}<$ $0.001)$.

Twelve patients subsequently underwent surgical exploration, with 10 subsequently undergoing curative resection (Table 3). R0 resections were achieved in all 10 patients, with nine also undergoing resection of one or more blood vessels, including six who underwent resection of the superior mesenteric or portal vein and five who underwent resection of the hepatic or celiac artery. The primary location of cancer was the head of the pancreas (five cases), the body $(n=4)$, the body and tail $(\mathrm{n}=1)$. The changes in CA 19-9 level before surgery ranged from $-98 \%$ to $-35 \%$. At the time of data analysis, only one patient (No. 19) with no residual tumor remained alive after 41.9 months of follow-up. The median PFS and OS of all patients in the present study were 10.1 months and 13.1 months, respectively (Fig. 3). The median OS was significantly longer in patients who underwent curative resection than in those that did not (16.8 months vs. 11 months, $\mathrm{p}<$ $0.01)$.

Of 33 patients completing the entire treatment protocol, 31 (93.9\%) experienced tumor progression. For five patients $(16.1 \%)$, tumor progression was locoregional, while for 23 $(74.2 \%)$, it was metastatic, and for three $(10.0 \%)$, it was of an unknown type. Chemotherapy was reintroduced in 20 patients $(64.5 \%)$ after protocol completion. The most common reason for no subsequent chemotherapy was poor PS. Gemcitabine plus erlotinib was used in 10 patients as the second line chemotherapy, while gemcitabine alone was used in five patients, TS-1 was used in three patients and gemcitabine plus cisplatin and capecitabine were administered to one patient each.

\section{Biomarker evaluation}

The median cfDNA concentration, KRAS mutant concentration and KRAS mutant fractional abundance before treatment were $108.5 \mathrm{ng} / \mathrm{mL}$ (IQR, 24.7 to $303 \mathrm{ng} / \mathrm{mL}$ ), 0.34 copies $/ \mu \mathrm{L}$ (IQR, 0 to 1.1 copies $/ \mu \mathrm{L}$ ), and $0.14 \%$ (IQR, $0 \%$ to $0.7 \%)$, respectively. After treatment, the median cfDNA concentration, KRAS mutant concentration and KRAS mutant fractional abundance were $18.4 \mathrm{ng} / \mathrm{mL}$ (IQR, 4.3 to 66 $\mathrm{ng} / \mathrm{mL}$ ), 0.21 copies $/ \mu \mathrm{L}$ (IQR, 0 to 5.1 copies $/ \mu \mathrm{L}$ ), and $0.09 \%$ (IQR, $0 \%$ to $0.77 \%$ ), respectively. The concentration of cfDNA was significantly lower after than before treatment $(\mathrm{p}<0.001)$ (Fig. 4A). In contrast, the KRAS mutant concentration and fractional abundance did not differ significantly before and after treatment (Fig. 4B and C). OS and PFS were not related to cfDNA concentration, KRAS mutation concentration or fractional abundance (S1 Fig.). 


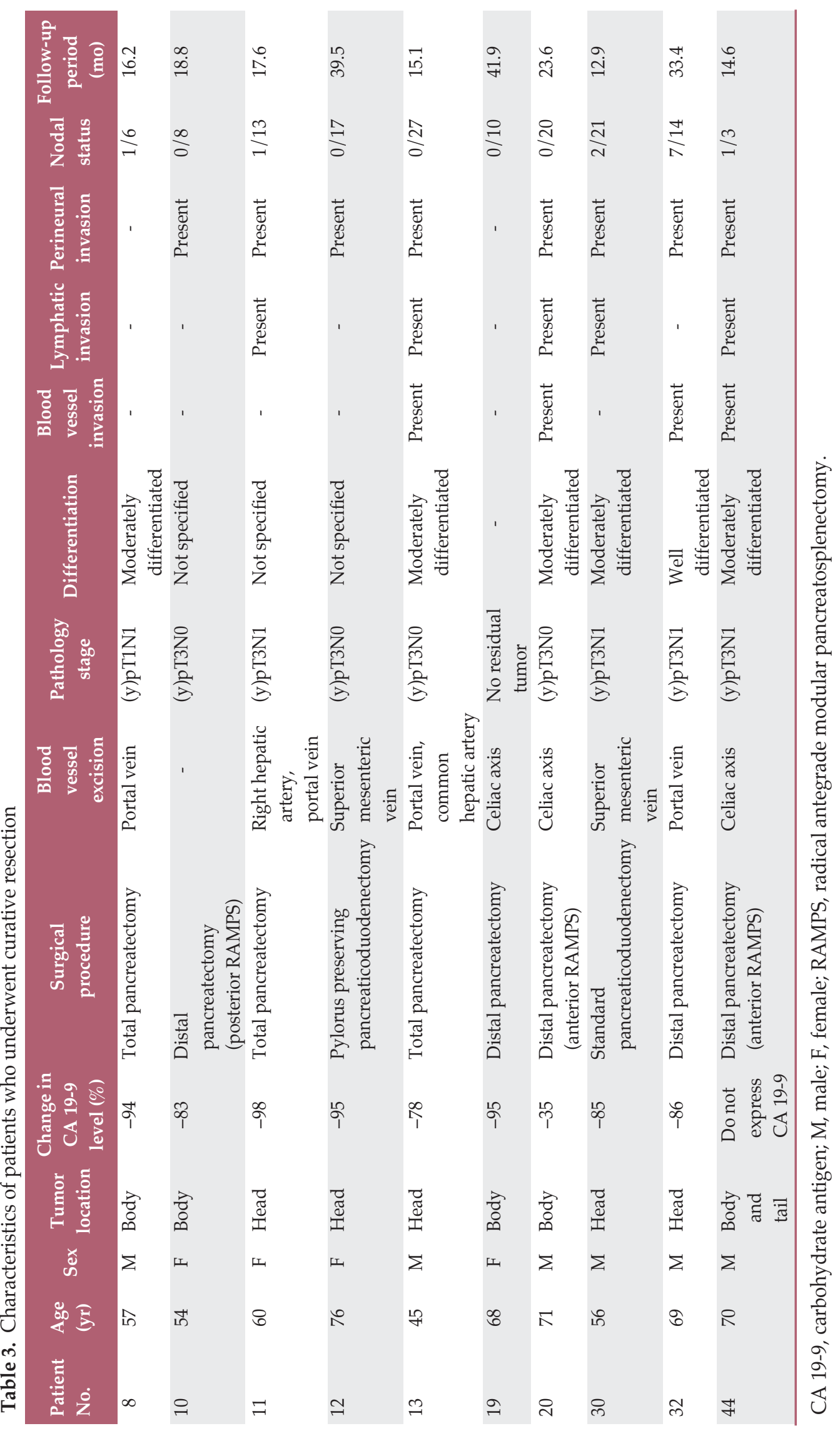



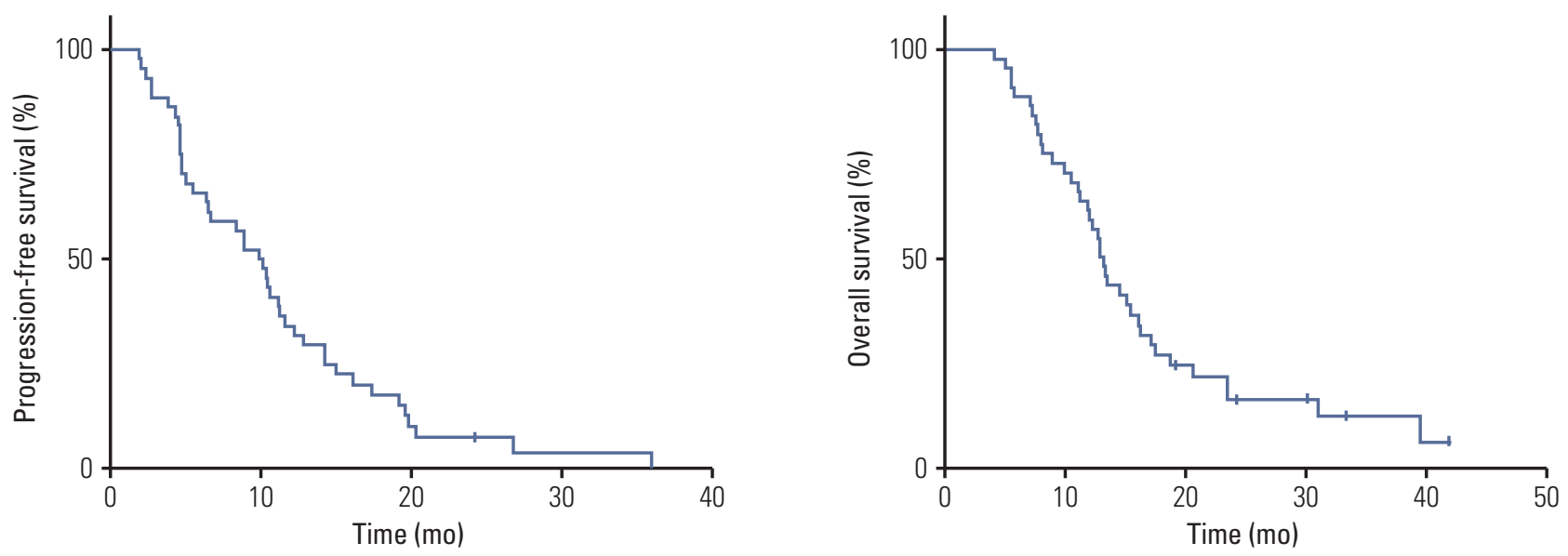

Fig. 3. Progression-free survival and overall survival of all patients.

\section{Discussion}

Most patients with pancreatic cancer present with metastatic or locally advanced tumors. Because of differences in natural history and therapy, clinical trials in patients with advanced pancreatic cancer have recently been subdivided into unresectable and metastatic disease [1]. The management of LAPC requires a multidisciplinary approach, with individual treatment based on careful assessment of tumor location, stage and resectability. The results of the LAP07 trial indicate that CRT does not add a survival advantage to chemotherapy alone [13]. However, responses to both chemotherapy and radiation vary among patients, making early identification of candidates for combined modality treatment essential. Moreover, the role of RT continues to be poorly defined, in part because of adherence to guidelines for the definition of target volume and technical details in treatment planning, although most protocol violations were minor and adherence appears to have been more carefully monitored than in prior trials.

Feasibility and compliance were the primary endpoints of this trial because it was a preliminary investigation designed gain specific information vital to planning subsequent studies. The null hypothesis set the true compliance rate of patients eligible for SIB-IMRT at $\leq 60 \%$. Overall, 33 of the 44 patients $(75 \%)$ completed the entire treatment protocol, a significantly higher proportion than the $60 \%$ expected $(\mathrm{p}=0.028)$. Given the feasibility and compliance of this treatment proto$\mathrm{col}$, induction chemotherapy with gemcitabine and cisplatin followed by concurrent SIB-IMRT is worthy of more rigorous evaluation in LAPC patients through randomized controlled studies.
Although more expensive than conventional RT, IMRT may reduce treatment-related toxicities in patients with pancreatic cancer [16]. The predominant treatment-related toxicities, namely nausea/vomiting, diarrhea and late gastrointestinal toxicity, were significantly reduced in patients receiving IMRT, although there were no apparent differences in outcome measures. No Grade 3 or 4 gastrointestinal toxicities associated with SIB-IMRT were reported in the current study.

Patients enrolled in the present study were treated with gemcitabine $300 \mathrm{mg} / \mathrm{m}^{2}$ plus concurrent SIB-IMRT, with the most common toxicity being grade 3 neutropenia (24.2\%). Haematological toxicity was more likely to be related to the type of concurrent chemotherapy than the technique of RT. In a recent randomized phase II trial [7], more patients treated with gemcitabine than with capecitabine had grade 3-4 hematological and non-hematological adverse effects. A capecitabine-based regimen may be an alternative to a gemcitabine-based regimen in the context of SIB-IMRT.

In the LAP 07 trial, erlotinib did not provide any additional benefit in the treatment of LAPC $[13,18]$. Combination chemotherapy regimens (e.g., 5-fluorouracil, leucovorin, irinotecan, oxaliplatin, FOLFIRINOX, and gemcitabine/nabpaclitaxel) have improved OS in patients with metastatic disease. FOLFIRINOX is a powerful first-line regimen that leads to resectability in a substantial portion of patients with initially unresectable pancreatic cancer [19-21]. The observed favorable survival after FOLFIRINOX should be discussed with patients with LAPC and good PS [22]. The role of combination chemotherapy regimens in LAPC is currently being actively investigated [23].

Induction chemotherapy provides theoretical advantages over standard adjuvant therapy, including treatment of dis- 
$\varangle$

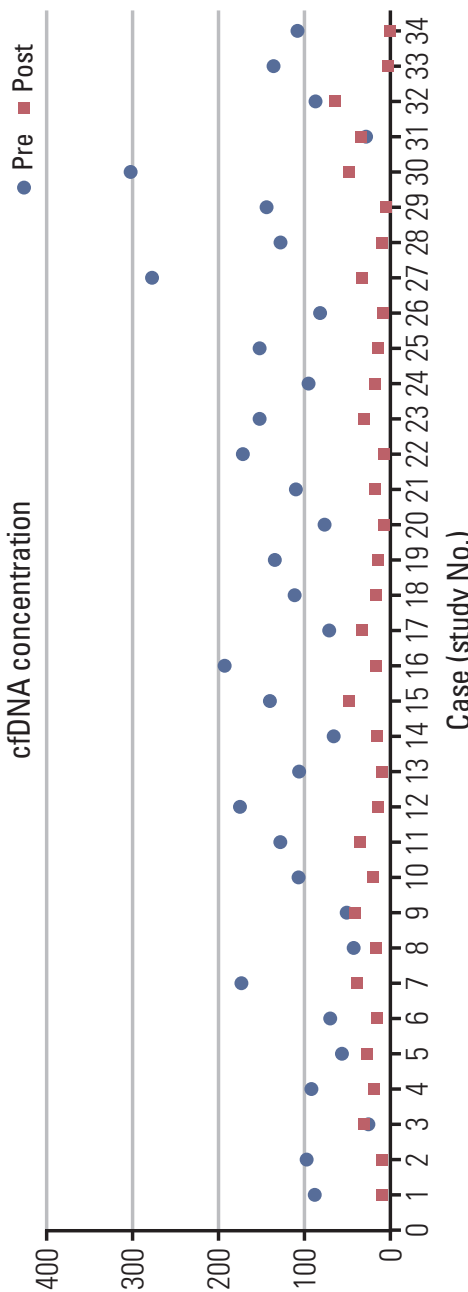

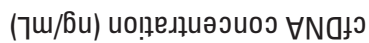

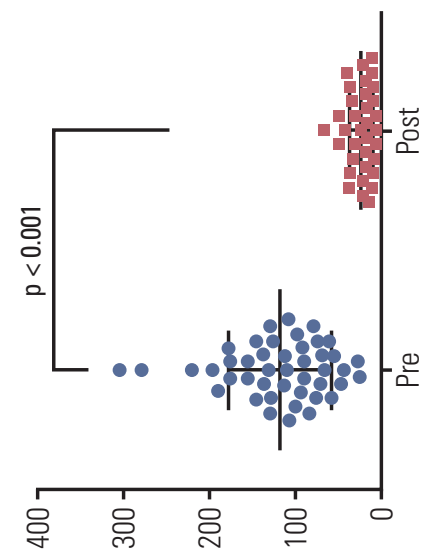

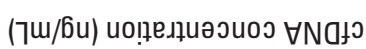

$\boldsymbol{\infty}$

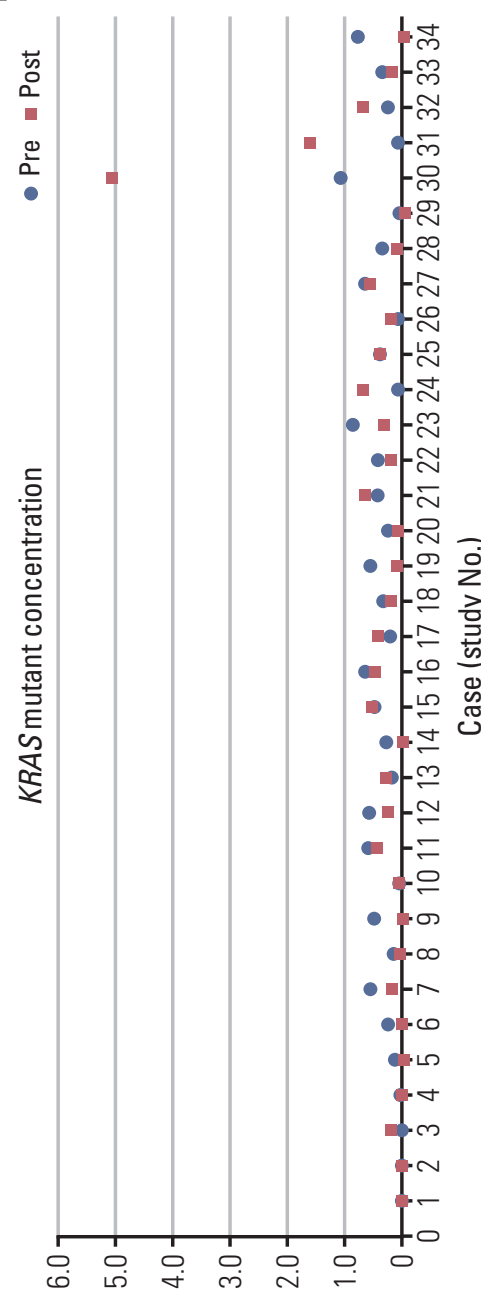

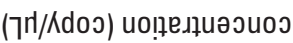
ұuęnu $S \forall y$ Y

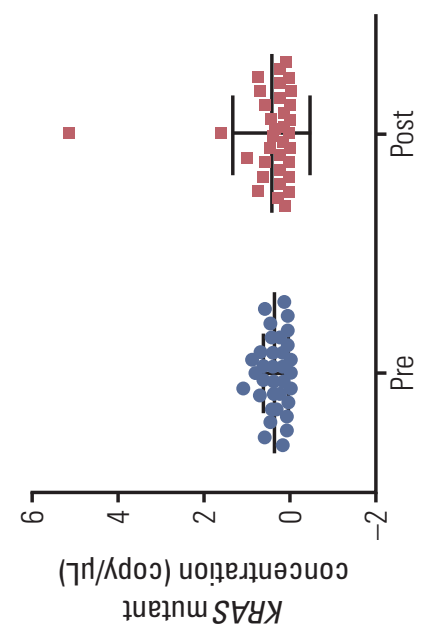

๑

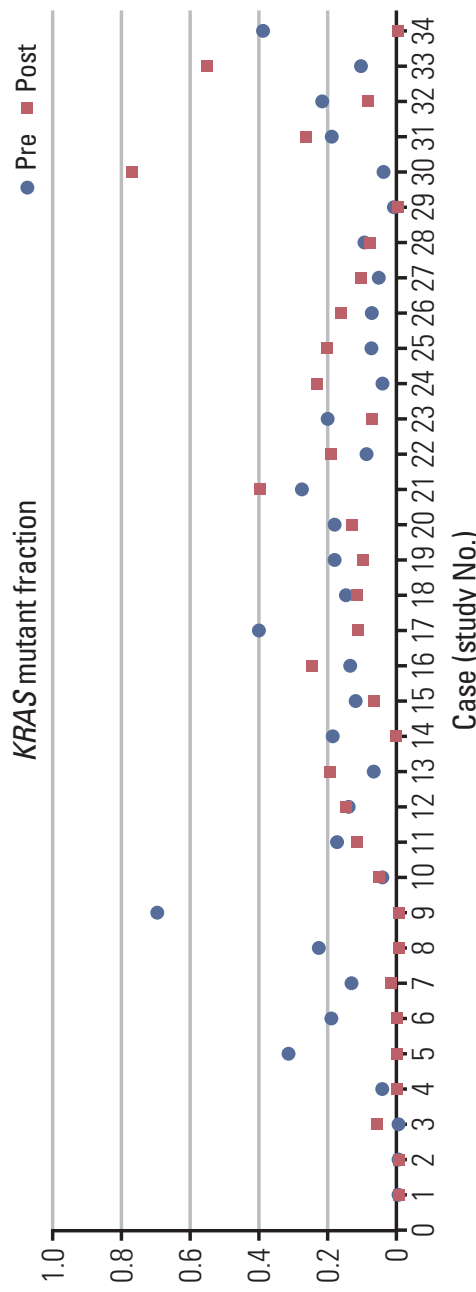

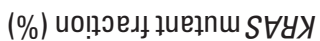


tant micrometastases, assessment of tumor response to treatment, and better selection of patients most appropriate for CRT or surgery. It has been hypothesized that extending the neoadjuvant period might improve the selection of patients who would benefit from the addition of RT [18]. In the present study, five patients $(11 \%)$ with early distant metastasis could avoid unnecessary CRT and $33(75 \%)$ completed the treatment regimen. Longer induction chemotherapy may facilitate selection of patients and sensitizing of tumors to subsequent CRT [24].

The frequency of a complete resection as well as long-term survival is low for patients with locally advanced pancreatic cancers. For example, the LAP07 trial demonstrated that only $4 \%$ of study participants responded to treatment sufficiently to enable pancreatectomy [13]. The proportion of patients who underwent resection after FOLFIRINOX for locally advanced pancreatic cancer ranged from $0 \%$ to $43 \%$ across studies [22]. Whether the use of more intensive initial induction chemotherapy might result in a better chance of significant tumor downstaging remains unclear. In the present study, 10 patients $(30 \%)$ underwent curative resection after the protocol was completed.

A recent study reported that the historical, cross-sectional imaging criteria for determining resectability are no longer appropriate in patients who receive FOLFIRINOX, with or without radiation therapy [21]. Current radiological imaging cannot distinguish between fibrosis and viable cancer, which is the basis for the continued imaging criteria for nonresectability. Despite reductions in CA 19-9 concentrations and/or tumor size, clear fat planes around critical vascular structures were not observed upon post-FOLFIRINOX preoperative imaging. A new biomarker is required to predict resectability and favorable response to curative resection.

The biology of LAPC is unique in that the tumor is confined locoregionally, without evidence of distant macrometastatic disease. No features have been found to clearly distinguish between tumors with a propensity to spread locally versus diffusely. The precise molecular mechanisms responsible for these behaviors are unclear [25]. Specific molecules involved in LAPC biology include transforming growth factor $\beta$, E-cadherin, $\mathrm{N}$-cadherin, the chemokine CXCL12 and Snail, along with K-ras [26-28].

Early identification of candidates for combined modality treatment is crucial. More than $90 \%$ of pancreatic ductal adenocarcinomas harbor somatic KRAS mutations, and pancreatic cancer biology may vary according to tumor-specific allelic ratio and dosage of mutated KRAS [29]. The presence of KRAS mutations was reported to adversely influence survival of patients with pancreatic cancer [30]. Low allelic ratios in tumor samples may be factors associated with good prognosis. A longer follow-up and evaluation of a greater number of patients will be necessary to confirm the role of
cfDNA.

It should be noted that this study was limited by the lack of a concurrent control arm. Because patients treated in prospective trials tend to have better outcomes than historical controls, a randomized design was considered. Ultimately, concerns regarding sample size, availability of patients with this rare disease entity, and, consequently, our ability to complete the trial in a timely fashion resulted in a single-arm design.

In summary, this trial met its stated goals, which were defined as the feasibility of and compliance with induction chemotherapy with gemcitabine and cisplatin followed by concurrent SIB-IMRT in patients with LAPC. The treatment regimen was well tolerated and active. Molecular markers are needed better predict responses to specific treatments, including radiation, and to allow more focused approaches to treatment selection. Further research assessing the role of circulating cfDNA as a biomarker in patients with LAPC is warranted.

\section{Electronic Supplementary Material}

Supplementary materials are available at Cancer Research and Treatment website (http:// www.e-crt.org).

\section{Conflicts of Interest}

This study was sponsored by Dong-A ST, Seoul, Korea (grant numbers 1240780-1).

\section{Acknowledgments}

The present study was partly supported by grants from the National Cancer Center, Goyang, Korea (grant numbers 1510202-2, 1610040-1, 1510203-2) and Dong-A ST, Seoul, Korea (grant numbers 1240780-1).

\section{Author Details}

${ }^{1}$ Center for Liver Cancer, National Cancer Center, Goyang, ${ }^{2}$ Department of System Cancer Science, Graduate School of Cancer Science and Policy, National Cancer Center, Goyang, ${ }^{3}$ Biometric Research Branch, Division of Cancer Epidemiology and Prevention, National Cancer Center, Goyang, ${ }^{4}$ College of Veterinary Medicine, Konkuk University, Seoul, ${ }^{5}$ Molecular Imaging and Therapy Branch, Research Institute National Cancer Center, Goyang, ${ }^{6}$ Emergency Department, National Cancer Center, Goyang, ${ }^{7}$ Department of Laboratory Medicine, Center for Diagnostic Oncology, National Cancer Center, Goyang, Korea 


\section{References}

1. Ryan DP, Hong TS, Bardeesy N. Pancreatic adenocarcinoma. N Engl J Med. 2014;371:2140-1.

2. Jung KW, Won YJ, Kong HJ, Oh CM, Cho H, Lee DH, et al. Cancer statistics in Korea: incidence, mortality, survival, and prevalence in 2012. Cancer Res Treat. 2015;47:127-41.

3. Chauffert B, Mornex F, Bonnetain F, Rougier P, Mariette C, Bouche $\mathrm{O}$, et al. Phase III trial comparing intensive induction chemoradiotherapy (60 Gy, infusional 5-FU and intermittent cisplatin) followed by maintenance gemcitabine with gemcitabine alone for locally advanced unresectable pancreatic cancer. Definitive results of the 2000-01 FFCD/SFRO study. Ann Oncol. 2008;19:1592-9.

4. Loehrer PJ Sr, Feng Y, Cardenes H, Wagner L, Brell JM, Cella $\mathrm{D}$, et al. Gemcitabine alone versus gemcitabine plus radiotherapy in patients with locally advanced pancreatic cancer: an Eastern Cooperative Oncology Group trial. J Clin Oncol. 2011; 29:4105-12.

5. Evans DB, George B, Tsai S. Non-metastatic pancreatic cancer: resectable, borderline resectable, and locally advanced-definitions of increasing importance for the optimal delivery of multimodality therapy. Ann Surg Oncol. 2015;22:3409-13.

6. Ambe C, Fulp W, Springett G, Hoffe S, Mahipal A. A Metaanalysis of randomized clinical trials of chemoradiation therapy in locally advanced pancreatic cancer. J Gastrointest Cancer. 2015;46:284-90.

7. Mukherjee S, Hurt CN, Bridgewater J, Falk S, Cummins S, Wasan $\mathrm{H}$, et al. Gemcitabine-based or capecitabine-based chemoradiotherapy for locally advanced pancreatic cancer (SCALOP): a multicentre, randomised, phase 2 trial. Lancet Oncol. 2013;14:317-26.

8. Colucci G, Giuliani F, Gebbia V, Biglietto M, Rabitti P, Uomo $\mathrm{G}$, et al. Gemcitabine alone or with cisplatin for the treatment of patients with locally advanced and/or metastatic pancreatic carcinoma: a prospective, randomized phase III study of the Gruppo Oncologia dell'Italia Meridionale. Cancer. 2002;94: 902-10.

9. Hu J, Zhao G, Wang HX, Tang L, Xu YC, Ma Y, et al. A metaanalysis of gemcitabine containing chemotherapy for locally advanced and metastatic pancreatic adenocarcinoma. J Hematol Oncol. 2011;4:11.

10. Ouyang G, Liu Z, Huang S, Li Q, Xiong L, Miao X, et al. Gemcitabine plus cisplatin versus gemcitabine alone in the treatment of pancreatic cancer: a meta-analysis. World J Surg Oncol. 2016;14:59.

11. Lundberg J, Reardon J, Blazer M, Phillips G, Bekaii-Saab T. Biweekly gemcitabine and low-dose cisplatin in the treatment of locally advanced or metastatic pancreatic cancer patients: a single institute experience. Med Oncol. 2016;33:4.

12. Kim JS, Lim JH, Kim JH, Im SA, Chie EK, Hwang JH, et al. Phase II clinical trial of induction chemotherapy with fixed dose rate gemcitabine and cisplatin followed by concurrent chemoradiotherapy with capecitabine for locally advanced pancreatic cancer. Cancer Chemother Pharmacol. 2012;70: 381-9.
13. Hammel P, Huguet F, van Laethem JL, Goldstein D, Glimelius B, Artru P, et al. Effect of chemoradiotherapy vs chemotherapy on survival in patients with locally advanced pancreatic cancer controlled after 4 months of gemcitabine with or without erlotinib: the LAP07 randomized clinical trial. JAMA. 2016; 315:1844-53.

14. Peixoto RD, Speers C, McGahan CE, Renouf DJ, Schaeffer DF, Kennecke HF. Prognostic factors and sites of metastasis in unresectable locally advanced pancreatic cancer. Cancer Med. 2015;4:1171-7.

15. Yovino S, Poppe M, Jabbour S, David V, Garofalo M, Pandya $\mathrm{N}$, et al. Intensity-modulated radiation therapy significantly improves acute gastrointestinal toxicity in pancreatic and ampullary cancers. Int J Radiat Oncol Biol Phys. 2011;79: 158-62.

16. Bittner MI, Grosu AL, Brunner TB. Comparison of toxicity after IMRT and 3D-conformal radiotherapy for patients with pancreatic cancer: a systematic review. Radiother Oncol. 2015;114:117-21.

17. Youl M, Hashem S, Brade A, Cummings B, Dawson LA, Gallinger $\mathrm{S}$, et al. Induction gemcitabine plus concurrent gemcitabine and radiotherapy for locally advanced unresectable or resected pancreatic cancer. Clin Oncol (R Coll Radiol). 2014;26:203-9.

18. Li D, O'Reilly EM. Adjuvant and neoadjuvant therapy for pancreatic cancer. Surg Oncol Clin N Am. 2016;25:311-26.

19. Nitsche U, Wenzel P, Siveke JT, Braren R, Holzapfel K, Schlitter AM, et al. Resectability after first-line FOLFIRINOX in initially unresectable locally advanced pancreatic cancer: a single-center experience. Ann Surg Oncol. 2015;22 Suppl 3:S1212-20.

20. Nanda RH, El-Rayes B, Maithel SK, Landry J. Neoadjuvant modified FOLFIRINOX and chemoradiation therapy for locally advanced pancreatic cancer improves resectability. J Surg Oncol. 2015;111:1028-34.

21. Ferrone CR, Marchegiani G, Hong TS, Ryan DP, Deshpande $\mathrm{V}, \mathrm{McDonnell} \mathrm{EI}$, et al. Radiological and surgical implications of neoadjuvant treatment with FOLFIRINOX for locally advanced and borderline resectable pancreatic cancer. Ann Surg. 2015;261:12-7.

22. Suker M, Beumer BR, Sadot E, Marthey L, Faris JE, Mellon EA, et al. FOLFIRINOX for locally advanced pancreatic cancer: a systematic review and patient-level meta-analysis. Lancet Oncol. 2016;17:801-10.

23. Shaib WL, Ip A, Cardona K, Alese OB, Maithel SK, Kooby D, et al. Contemporary management of borderline resectable and locally advanced unresectable pancreatic cancer. Oncologist. 2016;21:178-87.

24. Faisal F, Tsai HL, Blackford A, Olino K, Xia C, De Jesus-Acosta $A$, et al. Longer course of induction chemotherapy followed by chemoradiation favors better survival outcomes for patients with locally advanced pancreatic cancer. Am J Clin Oncol. 2016;39:18-26.

25. He J, Page AJ, Weiss M, Wolfgang CL, Herman JM, Pawlik 
TM. Management of borderline and locally advanced pancreatic cancer: where do we stand? World J Gastroenterol. 2014; 20:2255-66

26. Iacobuzio-Donahue CA, Fu B, Yachida S, Luo M, Abe H, Henderson CM, et al. DPC4 gene status of the primary carcinoma correlates with patterns of failure in patients with pancreatic cancer. J Clin Oncol. 2009;27:1806-13.

27. Chambers AF, Groom AC, MacDonald IC. Dissemination and growth of cancer cells in metastatic sites. Nat Rev Cancer. 2002;2:563-72.
28. Koenig A, Mueller C, Hasel C, Adler G, Menke A. Collagen type I induces disruption of E-cadherin-mediated cell-cell contacts and promotes proliferation of pancreatic carcinoma cells. Cancer Res. 2006;66:4662-71.

29. Lennerz JK, Stenzinger A. Allelic ratio of KRAS mutations in pancreatic cancer. Oncologist. 2015;20:e8-9.

30. Kwon MJ, Jeon JY, Park HR, Nam ES, Cho SJ, Shin HS, et al. Low frequency of KRAS mutation in pancreatic ductal adenocarcinomas in Korean patients and its prognostic value. Pancreas. 2015;44:484-92. 